\title{
Bioanalysis
}

\section{High-throughput method for the quantitation of metabolites and co-factors from homocysteine-methionine cycle for nutritional status assessment}

\begin{abstract}
Aim: There is increasing interest in the profiling and quantitation of methionine pathway metabolites for health management research. Currently, several analytical approaches are required to cover metabolites and co-factors. Results: We report the development and the validation of a method for the simultaneous detection and quantitation of 13 metabolites in red blood cells. The method, validated in a cohort of healthy human volunteers, shows a high level of accuracy and reproducibility. Conclusion: This high-throughput protocol provides a robust coverage of central metabolites and co-factors in one single analysis and in a high-throughput fashion. In large-scale clinical settings, the use of such an approach will significantly advance the field of nutritional research in health and disease.
\end{abstract}

First draft submitted: 3 May 2016; Accepted for publication: 27 July 2016; Published online: 25 August 2016

Keywords: high throughput $\bullet$ methionine pathway $\bullet$ one-carbon metabolism $\bullet$ red blood cells • UPLC-MS/MS

The one-carbon pathway, its metabolites and related B-vitamins co-factors (B6, B12 and B2) are receiving renewed interest because of the role of this metabolic cycle in epigenetic regulation of gene expression, development, in aging from birth to senescence, in several chronic conditions and in cancer [1-4]. One-carbon metabolism (1C) is a cyclic pathway whose essential components include sulfur-containing amino acids (AAs) such as methionine, water soluble $\mathrm{B}$-vitamins and cobalamin (vitamin B12) co-factordependent remethylation reaction, which cycles homocysteine to methionine. Most of the available carbon units are either provided by dietary methionine or choline [5-7]. Diet has been linked to specific modulations of the concentrations of $S$-adenosylmethionine (SAM), S-adenosylhomocysteine (SAH) and homocysteine (HCy) [3]. Furthermore, SAM is the donor of methyl groups for a large range of methyltransferase processes involved in a various metabolic and signal- ing pathways [8], RNA, proteins and DNA. The transfer of the methyl group produces $\mathrm{SAH}$, which is then metabolized to homocysteine. Other dietary components such as choline/betaine are an important source of one-carbon units, and contribute to methyltransferase activity via the betaine homocysteine methyl transferase enzymatic reactions. From a nutritional perspective, monitoring the activity of this $1 \mathrm{C}$ pathway in response to nutrition through metabolomics analysis (i.e., nutritional metabolomics) would generate a more comprehensive understanding of the interplay between host, environment and nutrient interaction.

Most blood-derived biomarkers of onecarbon metabolism exhibit adequate preanalytical stability and within-person reproducibility which allows their use as surrogates for the assessment of nutritional status in humans [9]. Epidemiological and intervention studies linking diet with epigenomic regulation have generated an interest in quantita-
Laeticia Da Silva', Sebastiano Collino', Ornella Cominetti', Francois-Pierre Martin', Ivan Montoliu', Sergio Oller Moreno', John Corthesy', Jim Kaput', Martin Kussmann', Jacqueline Pontes Monteiro ${ }^{2}$ \& Seu Ping Guiraud ${ }^{*}, 1$ ${ }^{1}$ Nestlé Institute of Health Sciences SA, Campus EPFL, Innovation Quarter, $\mathrm{CH}-1015$ Lausanne, Switzerland ${ }^{2}$ Department of Pediatrics, Faculty of Medicine, Nutrition \& Metabolism, University of São Paulo, Bandeirantes Avenue, HCFMRP Campus USP, 3900, Ribeirão Preto, SP 14049-900, Brazil *Author for correspondence: seuping.guiraud@rd.nestle.com 
tively monitoring this metabolic pathway in a holistic fashion and with a single, high-throughput method compatible with clinical research. Over the last decade, mass-spectrometry-based metabolomics methods have demonstrated robust, accurate and precise quantitation of several one-carbon biomarkers in diverse biological matrices [10-13]. Metabolic profiling and metabolomics refer to comprehensive or preselective screening quantitation of low-molecular-weight compounds, sampled from specific tissues or biological fluids such as blood, urine or blood cells [14-16]. Most available analytical methods for methionine pathway metabolites have so far deployed multiple, and typically separated chromatographic, derivatization and/or detection schemes to identify and quantify methionine, homocysteine and cysteine, SAM/SAH and B vitamins [17-21]. To our knowledge, no global metabolomics approach exists to map the entire methionine pathway (Figure 1) in one integrated method and a few analytical methods have been developed and validated in red blood cells (RBC, erythrocytes) with limited analysis to SAM, $\mathrm{SAH}$ and $\mathrm{HCy}$ [22]. RBCs are preferred because whole blood contains metabolites released from any cell or tissue in the body, which cannot be accounted during postmeasurement analysis.

In order to partially address this challenge in human metabolic research and fill this major gap in capturing, in one single analysis, the whole dynamics of this complex node, a low-volume, quantitative LC-MS/MS method was developed. We report the use of this targeted and integrated approach to quantify 13 biochemical species in this pathway, with emphasis on folate/methionine cycles as illustrated in Figure 1. The method was validated in RBC, where levels of some of the quantified metabolites are more abundant compared with plasma [3], using stable isotope-labeled related compounds as internal standards (IS). The 13 compounds are homocysteic acid, taurine, serine, cysteine, glycine, homocysteine, riboflavin, methionine, pyridoxine, cystathionine, pyridoxamine, $\mathrm{SAH}$ and SAM. In this report, we describe the simultaneous measurement of these 13 compounds unbegged in the $1 \mathrm{C}$ pathway, which method will considerably increase the laboratory's efficiency compared with multiple assays and offer a simple, reliable and cost-effective method not only for research work but also for nutritional and clinical assessment applied to routine clinical samples.

\section{Experimental \\ Materials}

LC-MS grade acetonitrile (ACN), LC-grade methanol, formic acid, trichloroacetic acid (TCA), perfluoroheptanoic acid (PFHA), ascorbic acid, sodium hydroxide $0.1 \mathrm{M}(\mathrm{NaOH} 0.1 \mathrm{M})$, hydrochloric acid ( $\mathrm{HCl})$, dithio-

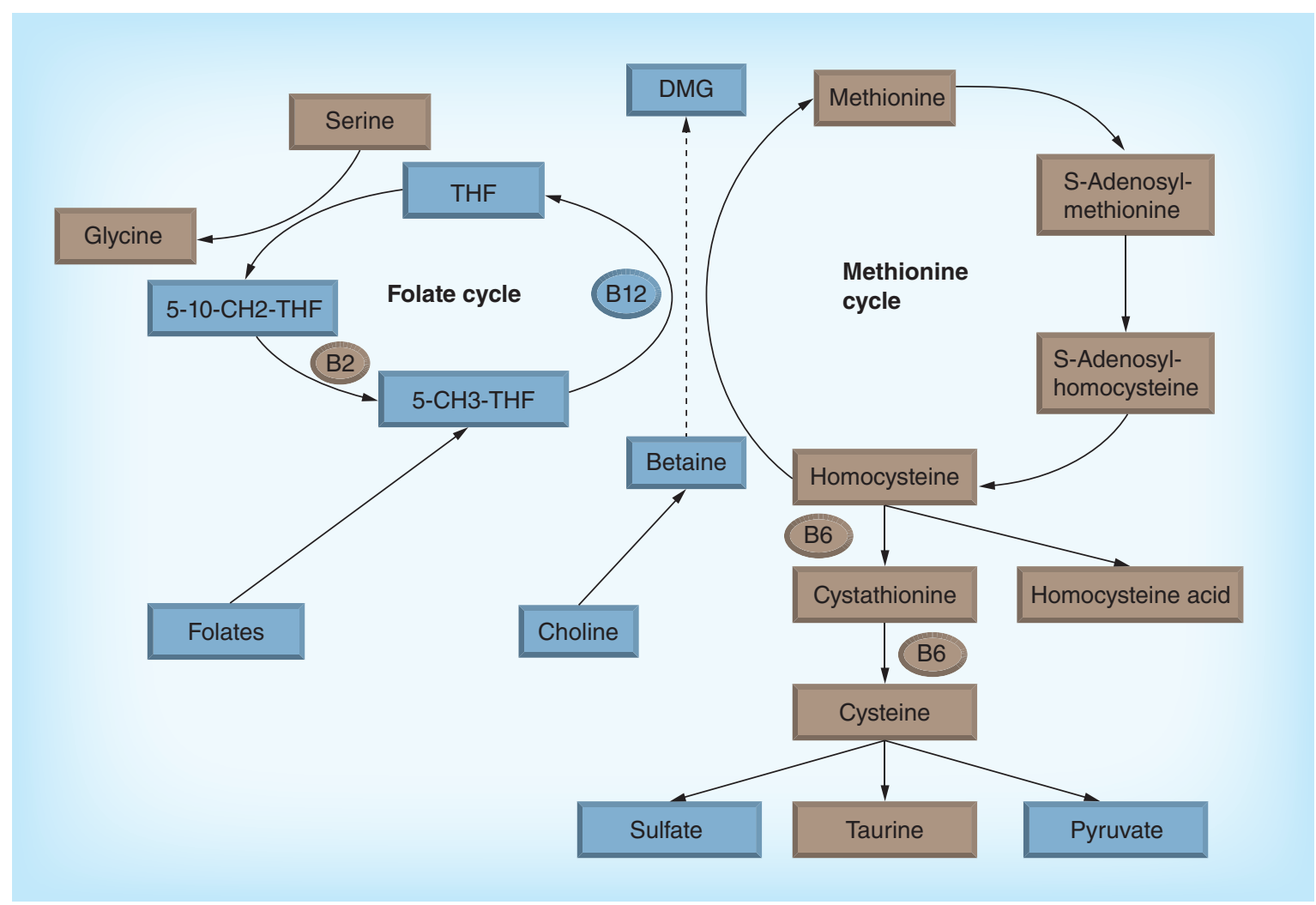

Figure 1. Metabolic pathway involving the measured (brown) metabolites from homocysteine-methionine cycle. 
threitol and ammonium acetate were purchased from Sigma-Aldrich Chemie GmbH (Buchs, Switzerland). Deionized water $(\mathrm{R}>18 \Omega \mathrm{M} / \mathrm{cm}$, TOC $<10 \mathrm{ppb})$ was used throughout experiments and produced by a Millipore-Q water system (Millipore, MA, USA). Homocysteic acid, taurine, serine, cysteine, glycine, $\mathrm{HCy}$, riboflavin, methionine, pyridoxine, cystathionine, $\mathrm{SAH}$, pyridoxamine, SAM, taurine ${ }_{-}^{13} \mathrm{C}_{2}$, glycine_d $\mathrm{d}_{2}$, riboflavin-dioxopyrimidine ${ }_{-}^{13} \mathrm{C}_{4}^{15} \mathrm{~N}_{2}$ and methionine_d $d_{3}$ standards were purchased from Sigma Aldrich Chemie GmbH. Homocysteic acid_d $\mathrm{d}_{4}$, serine- $\mathrm{d}_{3}$, pyridoxine_d $\mathrm{d}_{2}$, cystathionine_d $\mathrm{d}_{4}, S$-adenosylmethionine_d $d_{4}$ were purchased from CDN Isotopes (Quebec, Canada). Cysteine_d $\mathrm{d}_{2}$, homocysteine_d $\mathrm{d}_{4}$ and pyridoxamine_d $d_{3}$ were purchased from Cambridge Isotopes Laboratories (MA, USA). S-adenosylhomocysteine_d was purchased from Cayman Chemical (MI, USA).

For method development, ZIC-HILIC $3.5 \mu \mathrm{m}$, $100 \times 2.1 \mathrm{~mm}$ was purchased from Merck (Schaffhausen, Switzerland), Kinetex C18 $1.7 \mu \mathrm{m}, 2.1 \times 100 \mathrm{~mm}$ and Luna HILIC $3.5 \mu \mathrm{m}, 2 \times 150 \mathrm{~mm}$ were purchased from Phenomenex (CA, USA), Acquity BEH HILIC $1.7 \mu \mathrm{m}, 2.1 \times 100 \mathrm{~mm}$, Acquity BEH C18 $1.7 \mu \mathrm{m}$, $2.1 \times 100 \mathrm{~mm}$ and XSelect HSST3 C18 $2.5 \mu \mathrm{m}, 2.1 \times$ $100 \mathrm{~mm}$ were purchased from Waters Corporation (MA, USA), Primesep $1003 \mu \mathrm{m}, 2.1 \times 100 \mathrm{~mm}$ was purchased from Sielc Technologies (IL, USA) and Hypersil Gold C18 $3 \mu \mathrm{m}, 1 \times 100 \mathrm{~mm}$ was purchased from Thermofisher Scientific, Inc. (MA, USA). The following ion pairing agents were purchased from SigmaAldrich Chemie GmbH (Buchs, Switzerland): heptafluorobutyric acid (HFBA), pentadecafluorooctanoic acid and PFHA.

\section{Individual standard solutions and IS solutions}

Standard solutions as listed in Supplementary Table 1 were first weighted and dissolved individually in $0.1 \mathrm{M}$ $\mathrm{HCl}$. Because of partial solubility of some compounds in this solution, various solvents were assessed to obtain the full solubility. Same dilution solvents were used for individual IS stock solutions. Standard stock solutions and IS stock solutions were kept at $-20^{\circ} \mathrm{C}$ for 3 months.

\section{Calibration curves \& quality control samples}

Calibration standards for human studies were prepared by dilution of standard stock solutions in ACN/ $\mathrm{H}_{2} \mathrm{O}(5 / 95 ; \mathrm{v} / \mathrm{v})$ to obtain eight calibration solutions (calibrants 1-8) in $\mu \mathrm{M}$ range for homocysteic acid, taurine, serine, cysteine, glycine, methionine, SAH and SAM; and in $\mathrm{nM}$ range for riboflavin, pyridoxine, cystathionine and pyridoxamine as in Supplementary Table 2. An IS working solution was prepared by dilution of IS stock solutions in $\mathrm{ACN} / \mathrm{H}_{2} \mathrm{O}(5 / 95 ; \mathrm{v} / \mathrm{v})$ (Supplementary Table 3).
Calibration samples were prepared with each batch of samples and injected at both the beginning and the end of the real-life sample sequence. About $50 \mu \mathrm{l}$ of each calibration solution were pipetted and transferred into a $1.5-\mathrm{ml}$ microcentrifuge tube. About $10 \mu \mathrm{l}$ of IS working solution were then added to these solutions. About $190 \mu \mathrm{l}$ of $\mathrm{ACN} / \mathrm{H}_{2} \mathrm{O}(5 / 95 ; \mathrm{v} / \mathrm{v})$ solution were added to the mixture. The tubes were placed on an autosampler rack and vortexed on a plate vortexer for $15 \mathrm{~min}$ at $1350 \mathrm{rpm}$. The solutions were transferred into an HPLC vial for LC-MS/MS analysis. Quality controls (QC) at two different concentrations were added to each batch of samples. The low-QC (at the same concentration as calibration level 3) and highQC samples (calibration level 6) were prepared using the same protocol as the calibration samples.

\section{LC-MS/MS}

Separation and analysis were performed on an Accela UHPLC 1250 Pump (Thermo Fisher Scientific, Inc., MA, USA) coupled to a TSQ Quantum Vantage triple quadrupole (Thermo Fisher Scientific, Inc.) equipped with a heated ESI source. The sample vials were maintained at $4^{\circ} \mathrm{C}$ in the thermostatic autosampler $(\mathrm{Pal}$ HTS-xt, CTC Analytics AG, Zwingen, Switzerland). Chromatographic separation was obtained using gradient elution on a reversed-phase UPLC XSelect HSST3 $2.5 \mu \mathrm{m}, 2.1 \times 100 \mathrm{~mm}$ column at room temperature at a flow rate of $400 \mu \mathrm{l} / \mathrm{min}$. Mobile phase A was constituted of water containing $5 \mathrm{mM}$ PFHA and mobile phase $B$ was composed of $A C N$. The following ramped gradient profile was used: 0 min $95 \%$ A; 0.5 min $95 \%$ A; $8 \min 5 \%$ A; $10 \min 5 \%$ A; $11 \min 95 \%$ A; and 13 $\min 95 \% \mathrm{~A}$. The injection volume was $10 \mu \mathrm{l}$ and the total run time of analysis was $13 \mathrm{~min}$. All MS parameters were optimized by direct infusion of each individual standard. MS detection was performed using positive electrospray ionization $\left(\mathrm{ESI}^{+}\right)$operating at unit resolution. Electrospray voltage was set at $4 \mathrm{kV}$, sheath gas at 10 arbitrary units, auxiliary gas at 5 arbitrary units, vaporizer temperature at $200^{\circ} \mathrm{C}$ and capillary temperature at $350^{\circ} \mathrm{C}$. The collision gas (argon) was set at 1.5 mTorr. Single reaction monitoring (SRM) transitions and settings are given in Table 1. Automated tuned s-lens parameters were used and the scan time was set at $0.02 \mathrm{~s}$. Xcalibur 2.1 software was used for instrument control, data acquisition and data analysis.

\section{Human sample collection for method validation}

The human cohort for biological validation was comprised of 212 children (113 girls and 99 boys) between 9 and 13 years of age, clinically stable, studying at schools in Ribeirão Preto, Brazil. The blood 


\begin{tabular}{|c|c|c|c|c|c|}
\hline \multirow[t]{2}{*}{ Analyte } & \multirow{2}{*}{$\begin{array}{l}\text { Retention time } \\
\text { (min) }\end{array}$} & \multicolumn{2}{|c|}{ Quantitative transition } & \multicolumn{2}{|c|}{ Qualitative transition } \\
\hline & & SRM1 & CE (eV) & SRM2 & CE (eV) \\
\hline Glycine & 1.4 & $76-->30$ & 11 & - & - \\
\hline Glycine_d 2 & 1.4 & $78-->32$ & 7 & - & - \\
\hline Serine & 1.15 & $106-->60$ & 10 & $106-->42$ & 24 \\
\hline Serine- $d_{3}$ & 1.15 & $109-->63$ & 11 & & \\
\hline Cysteine & 1.4 & $122-->59$ & 20 & $122-->76$ & 11 \\
\hline Cysteine_d $\mathrm{d}_{2}$ & 1.4 & $124-->61$ & 23 & & \\
\hline Taurine & 0.6 & $126-->108$ & 11 & $126-->44$ & 18 \\
\hline Taurine ${ }_{-}{ }^{13} \mathrm{C}_{2}$ & 0.6 & $128-->110$ & 11 & & \\
\hline Homocysteine & 2.85 & $136-->90$ & 10 & $136-->56$ & 18 \\
\hline Homocysteine_d ${ }_{4}$ & 2.85 & $140-->94$ & 10 & & \\
\hline Methionine & 4.3 & $150-->104$ & 10 & $150-->56$ & 18 \\
\hline Methionine_d $d_{3}$ & 4.3 & $153-->107$ & 9 & & \\
\hline Pyridoxine & 4.5 & $170-->134$ & 18 & $170-->152$ & 18 \\
\hline Pyridoxine_d ${ }_{2}$ & 4.5 & $172-->136$ & 19 & & \\
\hline Pyridoxamine & 5.75 & $169-->152$ & 11 & $169-->134$ & 20 \\
\hline Pyridoxamine_d 3 & 5.75 & $172-->155$ & 12 & & \\
\hline Homocysteic acid & 0.6 & $184-->138$ & 10 & $184-->56$ & 18 \\
\hline Homocysteic acid_d ${ }_{4}$ & 0.6 & $188-->142$ & 9 & & \\
\hline Cystathionine & 5.2 & $223-->134$ & 13 & $223-->88$ & 29 \\
\hline Cystathionine_d $d_{4}$ & 5.2 & $227-->138$ & 13 & & \\
\hline Riboflavin & 3.4 & $377-->243$ & 15 & $377-->172$ & 28 \\
\hline Riboflavin ${ }_{-}^{13} \mathrm{C}_{4^{\prime}}{ }^{15} \mathrm{~N}_{2}$ & 3.4 & $383-->249$ & 13 & & \\
\hline SAH & 5.6 & $385-->134$ & 21 & $385-->136$ & 23 \\
\hline SAH_d ${ }_{4}$ & 5.6 & $389-->136$ & 18 & & \\
\hline SAM & 5.9 & $399-->250$ & 14 & $399-->136$ & 27 \\
\hline SAM_d ${ }_{3}$ & 5.9 & $402-->250$ & 13 & & \\
\hline
\end{tabular}

samples were obtained in 2013 and in 2014, and were the baseline of a supplementation intervention study (Harmonized Micronutrient Project, ClinTrials.gov identifier NCT01823744). Out of the total 212 children, 66 participated in both years ( 40 girls and 26 boys) giving a total of 278 samples ( 153 from girls and 125 from boys). Weight was neither an inclusion or exclusion criterion. Participants received institutional review board (Ethics Committee) approved compensation, as well as breakfast and lunch during each data collection day. Children who met any of the following criteria were not eligible to participate: children who participated in a clinical trial on the previous 4 weeks from the beginning of the study, children who received any type of vitamin or mineral supplement, children under a supervised dietary restriction, children suffering a chronic disease which would interfere with data collection, children who had a measured axillary temperature higher than $37^{\circ} \mathrm{C}$ in the previous 15 days from blood collection or who had three or more episodes of liquid stools in the previous $24 \mathrm{~h}$. Blood samples $(24 \mathrm{ml})$ were collected from participants after an overnight fast including three 4-ml EDTA Vacutainer tubes. Samples were kept on ice. Samples were centrifuged within 30 min of collection; plasma, buffy coat and RBC were separated and stored at $-80^{\circ} \mathrm{C}$ until analysis. Demographic data, anthropometric measurements, cognitive and health status, clinical anamnesis and drug use were collected using a standard questionnaire. 

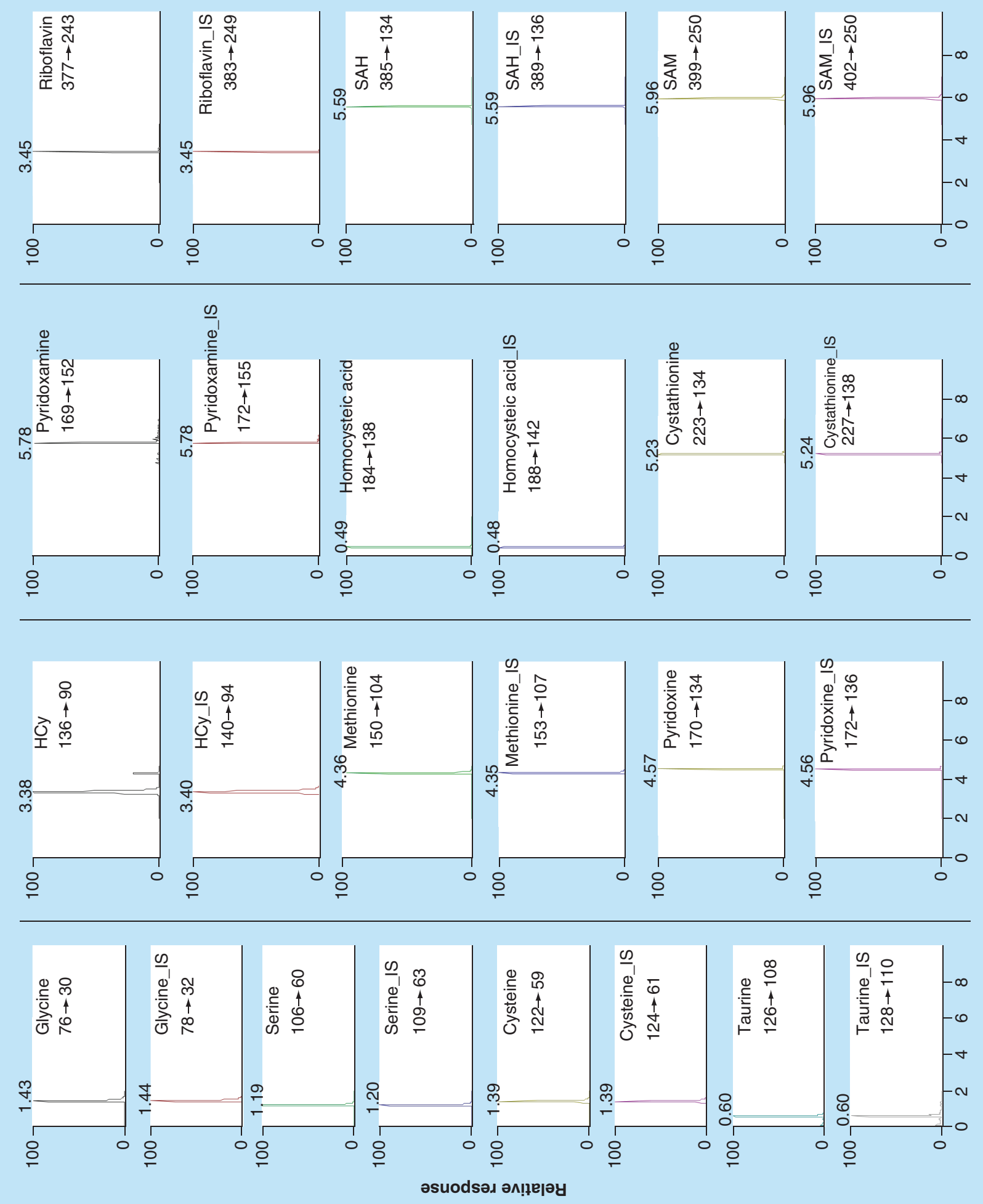


\section{RBC sample extraction}

$\mathrm{RBC}$ frozen samples were thawed and vortexed for $10 \mathrm{~s}$ and kept on ice. About $50 \mu \mathrm{l}$ of RBC samples were pipetted using a wide-bore tip and transferred into a $1.5-\mathrm{ml}$ microcentrifuge tube. About $10 \mu \mathrm{l}$ of IS working solution were then added to RBC samples. About $170 \mu \mathrm{l}$ of $0.1 \mathrm{M}$ sodium acetate solution and then $20 \mu \mathrm{l}$ of TCA $40 \%$ were added to the mixture. The tubes were vortexed for $30 \mathrm{~s}$ and left on ice for $30 \mathrm{~min}$ to allow protein precipitation. The tubes were then centrifuged at $14,500 \mathrm{rpm}$ for $10 \mathrm{~min}$ at $4^{\circ} \mathrm{C}$. The supernatants were pipetted and filtered through a $0.22-\mu \mathrm{m}$ filter and placed into HPLC vials and ready for LC-MS/MS analysis.

\section{Specificity \& selectivity}

We assessed retention time stability, chromatographic separation and MS/MS detection to determine specificity and selectivity of the method.

\section{LODs \& LOQs}

LODs and LOQs were evaluated on the basis of signal to noise ratio $(\mathrm{S} / \mathrm{N})$.

\section{Trueness, precision \& recovery}

To evaluate trueness and precision (repeatability and intermediate precision), spiked samples were analyzed at low, medium and high spiking levels in six replicates at each level. For repeatability, six replicates were prepared by the same operator on three separate occasions in a short period of time. For intermediate precision, the same protocol was followed with additional analyses conducted by two other different individuals on a total of six separated occasions.

\section{Matrix effects}

Quantitative measurement of matrix effects was determined by postextraction IS analyte spiking. Two sets of samples were prepared (five replicates for each set): one containing the IS analyte added to an extracted matrix (postextraction sample) and the other contained the IS analyte in the mobile phase solvent. Both sets of samples were spiked with the same concentration of IS analyte. Matrix effect values in \% were calculated using the following equation:

$$
\text { Matrix effect }(\%)=\mathrm{B} / \mathrm{A} \times 100
$$

where $\mathrm{A}$ is the mean of solvent solution peak area and $\mathrm{B}$ is the mean of post-extraction sample peak area.

The experiment was performed twice using two different IS concentrations. We report the mean of matrix effect values of both concentrations in Supplementary Table 4.

\section{Calibration curve \& QC}

Solvent and matrix-matched external calibration curves were compared. The former curves were used for quantitative determination. The calibration curves were constructed by calculating the peak area ratio of the analyte and IS for each analyte and concentration level. Linear regression analyses were performed using five replicates of the calibration curve data. The acceptance criterion for each backcalculated standard concentration was $\pm 15 \%$ deviation from the nominal value except at the lowest level, in other words, level 1 , which was set at $\pm 20 \%$.

\section{Statistical analysis}

Two-sample Kolmogorov-Smirnov tests were used to determine statistical significance between gender distributions of metabolites. p-values lower than 0.05 were considered as statistically significant. The statistical software R (version 3.2.2) [23] was used for such analyses and the package ggplot [ggplot2 Elegant Graphics for Data Analysis] was used for plotting distributions and scatterplots.

\section{Results \& discussion}

We developed and validated a method that allows the sensitive detection and robust quantitation of a range of biomolecules of diverse structures in the methionine cycle: vitamins, AAs, folates and some metabolites of those compounds.

\section{Optimization of method development}

Metabolite profiling in large-scale epidemiological studies requires fast, multianalyte methods for the measurement of various sets of small molecules related to specific biochemical pathways. Furthermore, the biggest difficulty of metabolomics analytical method development lies in the ability to cover diverse classes of metabolites. Thus, the main challenge of our LC-MS/MS method was to separate and analyze in the same run both polar and semipolar compounds of the 1C pathway (Figure 1). A first chromatographic separation test was performed on an Acquity BEH C18 column but displayed very poor retention for many compounds including glycine and taurine. To improve the retention of polar compounds, we assessed the use of ion-pairing agents, which are often used in smallmolecule analysis [24]. HFBA, pentadecafluorooctanoic acid and PFHA were tested, and PFHA providing the best compromises in terms of sensitivity (ion-pairing agents often cause ion suppression) and retention. HILIC columns (such as ZIC-HILIC, Luna HILIC and Acquity HILIC) were also tested to improve the retention of the most polar compounds. However, either peak tailing (for SAH, homocysteine and cys- 
tathionine) or low sensitivity (for taurine and SAH) was observed. An alternative Primesep 100 column was also assessed, which enables ion exchange and thus the retention of polar compounds without the addition of ion-pairing agents. However, this column had poor sensitivity for methionine and homocysteine. Different C18 columns were also assessed (Kinetex, Acquity BEH, Hypersil Gold, XSelect HSST3). The XSelect HSST3 showed the best response for most of the analytes. The ESI source in positive ion mode was the best ionization method for these semipolar and polar compounds. Automatic tuned s-lenses were applied and collision energy values were optimized for each transition. Typical SRM chromatograms are shown in Figure 2. Only one transition was found for glycine due to its low molecular weight. The sample preparation procedure was adapted from Wise et al. [22] with the following modifications: a 50- $\mu \mathrm{l}$ sample was used; $170 \mu \mathrm{l}$ of $0.1 \mathrm{M}$ sodium acetate solution and $20 \mu \mathrm{l}$ of TCA $40 \%$ were added; addition of $10 \mu \mathrm{l}$ IS solution; and no diethyl ether extraction. The method was optimized to minimize volume requirement for analysis. The use of diethyl ether to remove lipids and excessive TCA gave lower signal and wider peaks, especially for homocysteine and glycine, and was thus discarded. Removing this step resulted in a simplified extraction procedure that shortened preparation and analysis time.

\section{Method validation}

The method validation procedure here described was based on the guidelines issued by the European Medicines Agency and the industry for bioanalytical method validation.

\section{Selectivity}

Assessing whether a selected chromatographic peak is the analyte of interest (i.e., selectivity) was complicated by the level of endogenous analyte present in the biological matrix. We therefore used a combination of retention time and MS/MS detection to assess analyte selectivity in both solvent and RBC samples. The first SRM transition is used as quantifier and the other one is given as the qualifier (Table 1). Retention time of each analyte was compared with its IS analog. Each analytical series contained solvent blanks (to confirm the absence of interferences) in addition to the highest concentration of IS used for calibration curve determination. The selected method excluded false positives.

\section{Estimated LOD \& LOQ}

LOD and LOQ were evaluated on the basis of S/N; at least above 3 for the LOD and 10 for the LOQ by injecting RBC samples spiked with IS mixture of all analytes. Because of endogenous levels of some analytes and incomplete reference materials for all the analyzed metabolites, the LOD and the LOQ were determined by two different approaches. The first one consisted of spiking solvent with a series of diluted standard solutions and the second one consisted of RBC samples spiked with a series of diluted IS standard solutions [25] until reaching a signal to noise above 3 for the LOD and above 10 for the LOQ [26]. Indeed, stable isotopelabeled compounds have the same physicochemical properties and should therefore have the same analytical behavior than the analog molecules (e.g., retention time and intensity). Estimated LOD and LOQ values from RBC samples spiked with IS standard solutions are reported in Table 2 since they were most similar or had higher signals than with standard solutions in the solvent.

\section{Linearity of the calibration}

Calibration curves to quantify metabolites in biological fluids are generally prepared by spiking specific analytes in blank matrix. However, a blank matrix does not exist for quantifying endogenous metabolites. Matrices such as bovine serum albumin or solvent matrices are usually used to mimic the results expected with the real matrix. In the present method, the linearity was assessed in RBC matrices and the results were compared with those calculated with a solvent-based matrix.

Calibration curves were performed in both solvent and matrix (two different RBC origins were used). The

\begin{tabular}{|c|c|c|c|}
\hline Analyte & LOD & LOQ & Units \\
\hline Homocysteic acid & 0.25 & 0.5 & $\mu \mathrm{M}$ \\
\hline Taurine & 3.0 & 4.0 & $\mu \mathrm{M}$ \\
\hline Serine & 0.5 & 1.0 & $\mu \mathrm{M}$ \\
\hline Cysteine & 0.1 & 0.25 & $\mu \mathrm{M}$ \\
\hline Glycine & 0.5 & 1.0 & $\mu \mathrm{M}$ \\
\hline Homocysteine & 0.5 & 1.0 & $\mu \mathrm{M}$ \\
\hline Riboflavin & 3.0 & 4.0 & $\mathrm{nM}$ \\
\hline Methionine & 0.25 & 0.5 & $\mu \mathrm{M}$ \\
\hline Pyridoxine & 1.0 & 1.5 & $\mathrm{nM}$ \\
\hline Cystathionine & 2.0 & 3.0 & $\mathrm{nM}$ \\
\hline $\mathrm{SAH}$ & 0.025 & 0.05 & $\mu \mathrm{M}$ \\
\hline Pyridoxamine & 2.5 & 5.0 & $\mathrm{nM}$ \\
\hline SAM & 0.025 & 0.05 & $\mu \mathrm{M}$ \\
\hline
\end{tabular}


RBC calibration slopes differed by $<20 \%$ from the solvent-based calibration curves. Consequently, only solvent-based calibration curves were considered to accurately quantify these analytes in RBC samples during the method validation. The linearity of the calibration curves was assessed using eight concentrations with five replicates each. The relationship between analyte response and concentration was found linear in the solvent in the range described in Supplementary Table 2. No curvature pattern was observed for any analyte which allowed analysis using a linear regression. A weighting factor of $1 / \mathrm{x}$ was applied for all compounds to achieve a linear model with the lowest relative errors. All coefficients of linear regression $\left(r^{2}\right)$ were found above 0.990. Typical calibration curves obtained for methionine are shown in Figure 3.

\section{Trueness, precision \& recovery}

The real matrix was spiked at three levels (low, medium and high) with the standard solution to assess the trueness and precision of the method (expressed as a bias and recovery). However, high levels of some endogenous compounds interfere with the calculation of trueness and precision at the lowest spiking level. Hence,

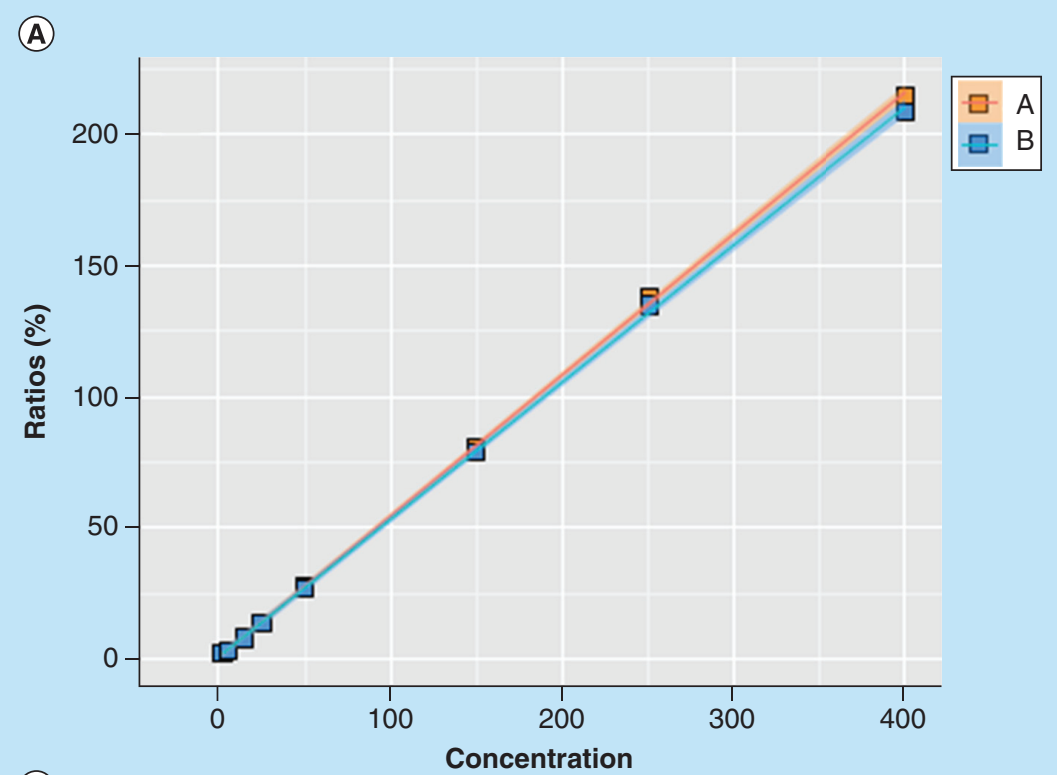

(B)

Concentration

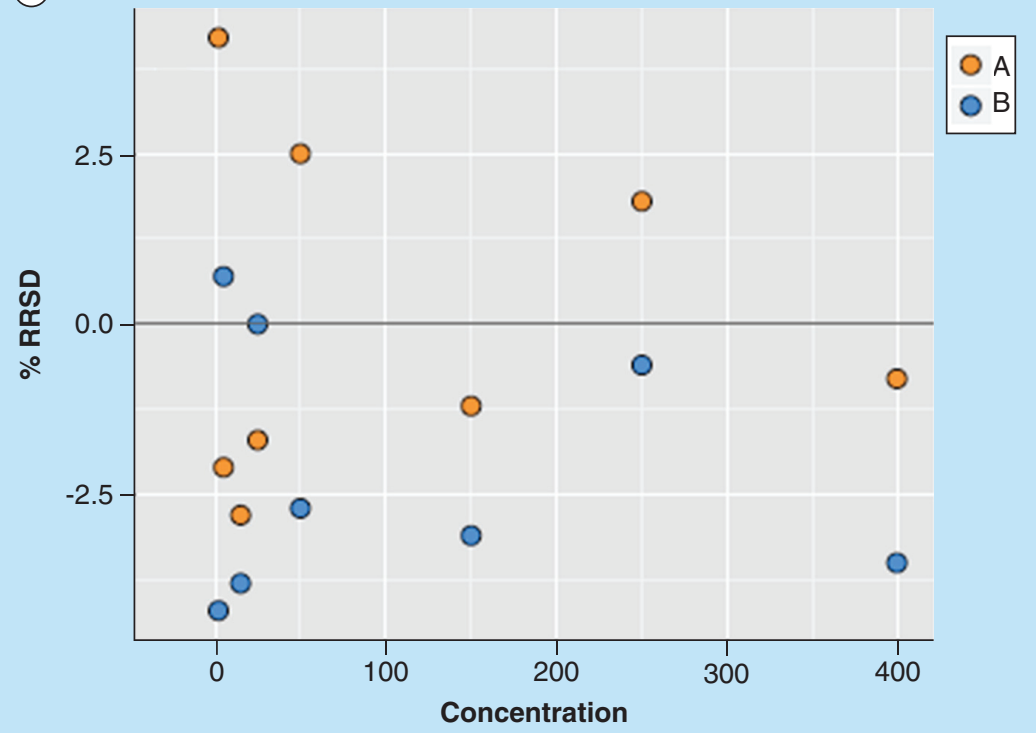

Figure 3. Example of a linear calibration curve (two datasets in a same run) with $1 / x$ weighting factor for methionine. (A) Area ratio against concentration. (B) Relative residual standard deviation against concentration. RRSD: Relative residual standard deviation. 
Table 3. Repeatability and intermediate trueness and precision values at three different concentration levels, in other words, low, high and medium levels ( $n=6$ per day, per level) to determine trueness and precision of the developed method in real matrix samples.

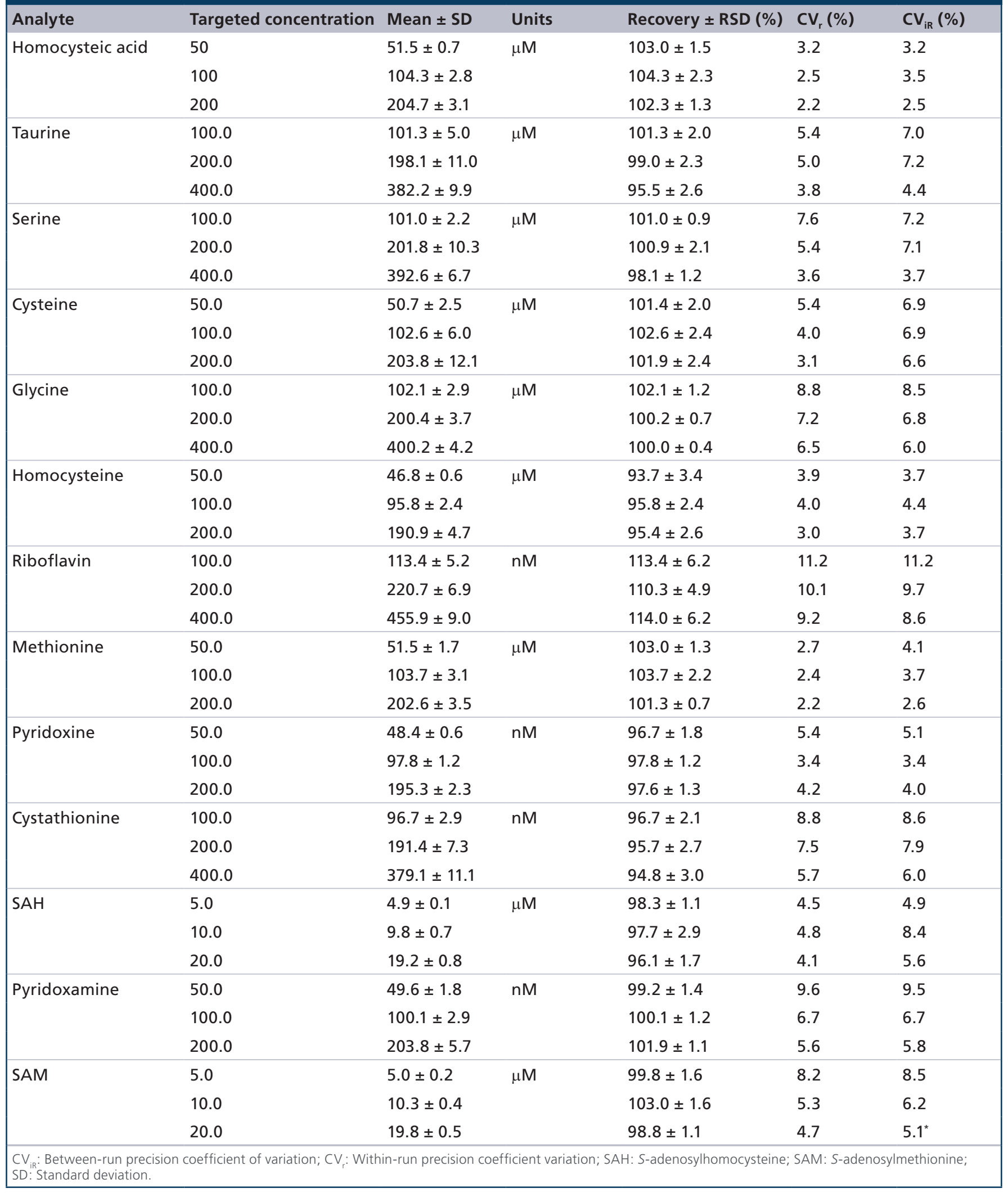


a much higher amount $(>10$-fold the recommended concentration for the lowest spiking level) was spiked into the sample:

$$
\text { Recovery } \%=100 \times \frac{\begin{array}{l}
\text { (Observed concentration }- \\
\text { endogenous concentration) }
\end{array}}{\text { Reference concentration }}
$$

Values for trueness and precision of spiked RBC samples are summarized in Table 3. Within-run precision (repeatability $\mathrm{r}$ ) and between-run precision (intermediate reproducibility $\mathrm{iR}$ ) coefficients of variation were found below $12 \%$ for all spiking levels, which are within the recommended limits. Recoveries were found between 93 and $114 \%$, which are also within the recommended limits.

\section{Matrix effects}

Matrix effects may occur in any LC-MS analysis by the presence of co-eluting compounds in the sample matrix, especially in complex matrices such as RBC lysates or plasma. The use of stable isotope-labeled related compounds as IS is an efficient and widely accepted approach to overcome and correct matrix effects with consequent improvements in determining trueness and precision of the methods. Ion suppression may occur when using ion pairing such as HFBA. Hence, we evaluated ion suppression even though stable isotope-labeled compounds were used in our method. Quantitative measurements for estimating the matrix effects are presented in Supplementary Table 4.

\section{Assessment \& validation of the method to human RBC samples}

The method was utilized to analyze these metabolites in RBC samples from a study of children aged from 9 to 13 . Previous studies extensively described how gender and age influence blood folate, vitamin B12, vitamin B6 and homocysteine levels in European adolescents $[27,28]$. Our data show also trends toward gender specificities for some metabolites in erythrocytes as shown in Figure 4. A previous study of 499 boys and 552 girls showed that plasma concentrations for homocysteine increased progressively with age, and remains higher in boys [28]. Interestingly, that study also reported that fortified breakfast intake is associated with the increased in vitamin B status and lower homocysteine concentration, which suggested that the supply of co-factors stimulated the metabolism of homocysteine resulting in lower levels in the circulation. The determination of AA concentrations in erythrocytes provided important additional information to the plasma AA levels in clinical conditions. In particular, little is known about sulfur-containing AA levels in erythrocytes. Sulfur-containing AAs may play a key role in glutathione metabolism and management of oxidative stress at the whole body level. Knowledge of the AA distribution between plasma and RBC may yield information for a better understanding of AA transport across the erythrocyte membrane and their metabolism in erythrocytes in childhood. In addition, it is well known that glutathione homeostasis, and therefore management of oxidative stress, is altered by dietary vitamin B6 deficiency.

\section{Conclusion}

Overall we have developed and fully validated a highthroughput LC-MS/MS method for the simultaneous detection and quantitation of 13 key metabolites of the folate/methionine pathways of the one-carbon cycle in RBC. Our method provides a novel analytical tool to capture in one run, and with high level of accuracy and reproducibility metabolic readouts associated with diet intake [29]. This pathway in RBCs may play a role in individual metabolic status or at least correlate with changes in nutrition. Our current observations suggest that gender influences the methionine-homocysteine pathway in RBC in childhood. Previous studies indicated that large proportion of free AAs were found in RBCs and these cells may be involved in interorgan AA transport [29-31] The one-carbon metabolite panel may help assessing individual nutritional requirements for healthy growth and development, where there are currently no available B-vitamin reference values for healthy adolescents. Differences between sexes may indicate a need for gender-specific nutritional management solutions.

\section{Future perspective}

This novel method provides a robust coverage of central metabolites and co-factors in one single analysis and in a high-throughput fashion. In large-scale clinical settings, the use of such an approach will contribute to the field of nutritional research in health and disease [32]. The analytical coverage of the method could be further extended to map the entire pathway, and therefore choline, betaine and dimethylglycine components of the cycle is currently missing. Folate metabolites such as 5-methyltetrahydrofolate would also be of utmost interest to appropriately cover the multiple metabolic pathways of $1 \mathrm{C}$ metabolism. To some extent, this analytical method in RBC samples could be adapted, following sample extraction optimi- 
(A)

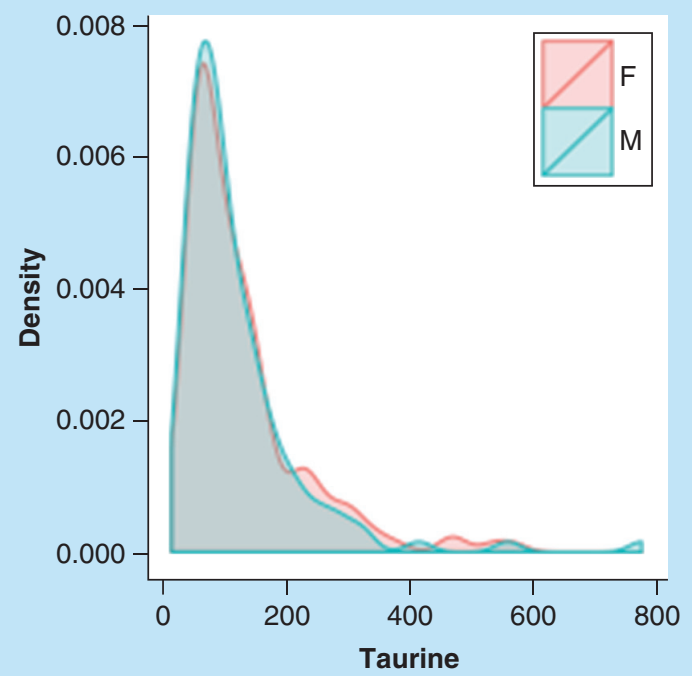

(c)

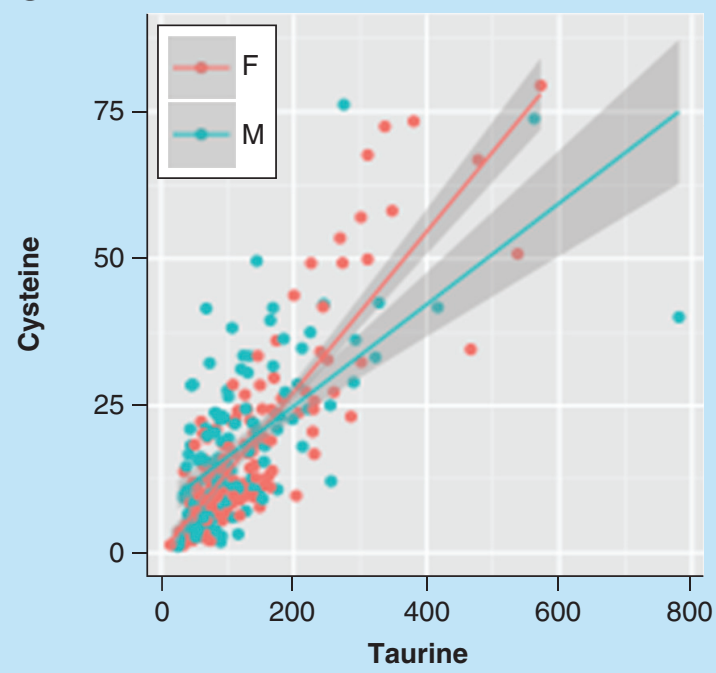

(B)

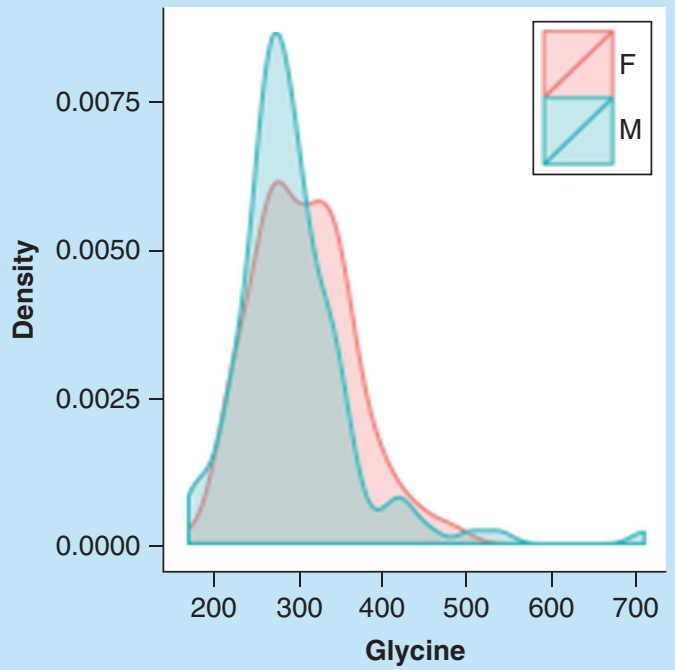

(D)

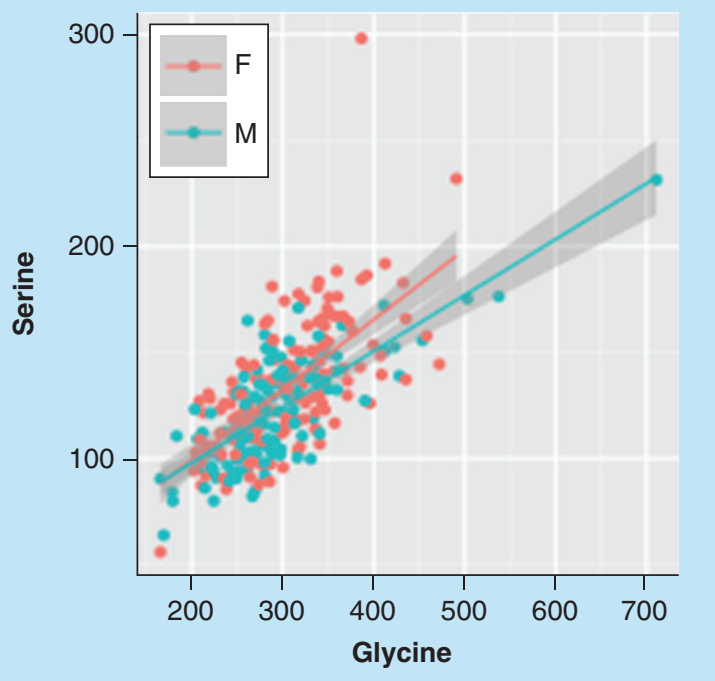

Figure 4. Gender differences. Density plots of (A) taurine and (B) glycine, and scatter plots showing high correlation between (C) cysteine and taurine and between (D) serine and glycine. The color codes in all four figures correspond to different genders: red depicts samples from female participants and green from male participants. Pearson correlation between cysteine and taurine for females is 0.85 and for males is 0.64 . Pearson correlation between serine and glycine for females is 0.65 and for males is 0.70 .

F: Female; M: Male.

zation, to other biofluids, such as plasma and cerebrospinal fluid, to further decipher nutrition-metabolic diseases mechanisms in the field of cardiometabolic and brain health [33]. The LC-MS settings could remain unchanged as LC-MS is known to be a sensitive technique to cover nanomolar range in matrices such as plasma and cerebrospinal fluid.

\section{Supplementary data}

To view the supplementary data that accompany this paper please visit the journal website at: www.future-science.com/ doi/full/10.4155/bio-2016-0112

\section{Acknowledgements}

The authors wish to thank L Dayon, R Faehndrich and AN Galindo for assistance during the method development and validation, and manuscript reviewing.

\section{Financial \& competing interests disclosure}

The authors have no relevant affiliations or financial involvement with any organization or entity with a financial interest in or financial conflict with the subject matter or materials discussed in the manuscript. This includes employment, consultancies, honoraria, stock ownership or options, expert testimony, grants or patents received or pending, or royalties. 
No writing assistance was utilized in the production of this manuscript.

\section{Ethical conduct of research}

The authors state that they have obtained all appropriate institution review board approvals or have followed the principles outlined in the Declaration of Helsinki for all human experimental investigations. In addition, for investigations involving human subjects, informed consent has been obtained from the participants involved. Children were included only if they had obtained their legal representative's informed consent and if they signed the Statement of Informed Assent for participants.

\section{Open access}

This work is licensed under the Attribution-NonCommercialNoDerivatives 4.0 Unported License. To view a copy of this license, visit http://creativecommons.org/licenses/by-ncnd/4.0/

\section{Executive summary}

\section{Background}

- Profiling and quantitation of methionine pathway metabolites for health management research is gaining considerable interest. Currently, several analytical approaches are required to cover metabolites and co-factors.

\section{Results}

- A new LC-MS/MS method for the simultaneous quantitation of 13 metabolites and co-factors of the methionine cycle in RBC is presented and provides comprehensive insights into the methionine metabolic network.

- The method is highly accurate and precise while requiring relatively low sample volume $(50 \mu \mathrm{l})$.

- A simple sample preparation and rapid chromatographic run time of $13 \mathrm{~min}$ ensure a high sample throughput.

- The method was validated in a cohort of healthy human volunteers and revealed a high level of accuracy and reproducibility and therefore suitable for other large-scale clinical studies.

\section{References}

Papers of special note have been highlighted as:

- of interest; $\bullet$ of considerable interest

1 Kaput J, Kussmann M, Radonjic M, Virgili F, Perozzi G. Human nutrition, environment, and health. Genes Nutr. 10(5), 489 (2015).

2 Monteiro JP, Kussmann M, Kaput J. The genomics of micronutrient requirements. Genes Nutr. 10(4), 466 (2015).

3 Monteiro JP, Wise C, Morine MJ et al. Methylation potential associated with diet, genotype, protein, and metabolite levels in the Delta Obesity Vitamin Study. Genes Nutr. 9(3), 403 (2014).

4 Andres E, Loukili NH, Noel E et al. Vitamin B12 (cobalamin) deficiency in elderly patients. CMAJ 171(3), 251-259 (2004).

5 Wadhwani NS, Pisal HR, Mehendale SS, Joshi SR. A prospective study of maternal fatty acids, micronutrients and homocysteine and their association with birth outcome. Matern. Child Nutr. 11(4) 559-573 (2015).

6 Stead LM, Brosnan JT, Brosnan ME, Vance DE, Jacobs RL. Is it time to reevaluate methyl balance in humans? Am. J. Clin. Nutr. 83(1), 5-10 (2006).

7 Rubio-Aliaga I, Roos B, Sailer M et al. Alterations in hepatic one-carbon metabolism and related pathways following a high-fat dietary intervention. Physiol. Genomics 43(8), 408-416 (2011).

8 Strain JJ, Dowey L, Ward M, Pentieva K, McNulty H. B-vitamins, homocysteine metabolism and CVD. Proc. Nutr. Soc. 63(4), 597-603 (2004).

9 Deac OM, Mills JL, Shane B et al. Tryptophan catabolism and vitamin B-6 status are affected by gender and lifestyle factors in healthy young adults. J. Nutr. 145(4), 701-707 (2015).

10 Field MS, Shields KS, Abarinov EV et al. Reduced MTHFD1 activity in male mice perturbs folate- and choline-dependent one-carbon metabolism as well as transsulfuration. J. Nutr. 143(1), 41-45 (2013).

11 Papakostas GI, Cassiello CF, Iovieno N. Folates and $S$ adenosylmethionine for major depressive disorder. Can. J. Psychiatry 57(7), 406-413 (2012).

12 Wernimont SM, Clark AG, Stover PJ et al. Folate network genetic variation predicts cardiovascular disease risk in nonHispanic white males. J. Nutr. 142(3), 1272-1279 (2012).

13 Mikael L. G, Pancer J, Wu Q, Rozen R. Disturbed onecarbon metabolism causing adverse reproductive outcomes in mice is associated with altered expression of apolipoprotein $\mathrm{AI}$ and inflammatory mediators PPARalpha, interferongamma, and interleukin-10. J. Nutr. 142(3), 411-418 (2012).

14 Martin FP, Collino S, Rezzi S. 1H NMR-based metabonomic applications to decipher gut microbial metabolic influence on mammalian health. Magn. Reson. Chem. 49 (Suppl. 1), S47-S54 (2011).

15 Collino S, Martin FP, Kochhar S, Rezzi S. Nutritional metabonomics: an approach to promote personalized health and wellness. Chimia (Aarau) 65(6), 396-399 (2011).

16 Collino S, Martin FP, Kochhar S, Rezzi S. Monitoring healthy metabolic trajectories with nutritional metabonomics. Nutrients 1(1), 101-110 (2009).

17 Kirsch SH, Herrmann W, Rabagny Y, Obeid R. Quantification of acetylcholine, choline, betaine, and dimethylglycine in human plasma and urine using stableisotope dilution ultra performance liquid chromatography- 
tandem mass spectrometry. J. Chromatogr. B Analyt. Technol. Biomed. Life Sci. 878(32), 3338-3344 (2010).

18 Ueland PM, Midttun O, Windelberg A, Svardal A, Skalevik R, Hustad S. Quantitative profiling of folate and one-carbon metabolism in large-scale epidemiological studies by mass spectrometry. Clin. Chem. Lab Med. 45(12), 1737-1745 (2007).

- Comprehensive measurement of folate and one-carbon metabolites in large-scale epidemiological studies.

19 Johansson M, Van GB, Vollset SE et al. One-carbon metabolism and prostate cancer risk: prospective investigation of seven circulating B vitamins and metabolites. Cancer Epidemiol. Biomarkers Prev. 18(5), 1538-1543 (2009).

20 Midttun O, Hustad S, Ueland PM. Quantitative profiling of biomarkers related to B-vitamin status, tryptophan metabolism and inflammation in human plasma by liquid chromatography/tandem mass spectrometry. Rapid Commun. Mass Spectrom. 23(9), 1371-1379 (2009).

21 Sigit JI, Hages M, Brensing KA et al. Total plasma homocysteine and related amino acids in end-stage renal disease (ESRD) patients measured by gas chromatographymass spectrometry - comparison with the Abbott IMx homocysteine assay and the HPLC method. Clin. Chem. Lab Med. 39(8), 681-690 (2001).

22 Wise CK, Cooney CA, Ali SF, Poirier LA. Measuring $S$-adenosylmethionine in whole blood, red blood cells and cultured cells using a fast preparation method and highperformance liquid chromatography. J. Chromatogr. B Biomed. Sci. Appl. 696(1), 145-152 (1997).

-. Is of considerable interest for its measurement of SAM in RBC.

23 R Development Core Team. R: A Language and Environment for Statistical Computing. The R Foundation for Statistical Computing, Vienna, Austria (2011).

24 Armstrong M, Jonscher K, Reisdorph NA. Analysis of 25 underivatized amino acids in human plasma using ionpairing reversed-phase liquid chromatography/time-of-flight mass spectrometry. Rapid Commun. Mass Spectrom. 21(16), 2717-2726 (2007).
25 Van de Merbel NC. Quantitative determination of endogenous compounds in biological samples using chromatographic techniques. Trends Anal. Chem. 27(10), 924-933 (2008).

26 Shrivastava A, Gupta BV. Methods for the determination of limit of detection and limit of quantitation of the analytical methods. Chron. Young Sci. 2, 21-25 (2011).

27 Kerr MA, Livingstone B, Bates CJ et al. Folate, related B vitamins, and homocysteine in childhood and adolescence: potential implications for disease risk in later life. Pediatrics 123(2), 627-635 (2009).

28 Gonzalez-Gross M, Benser J, Breidenassel C et al. Gender and age influence blood folate, vitamin B12, vitamin B6, and homocysteine levels in European adolescents: the Helena Study. Nutr. Res. 32(11), 817-826 (2012).

29 Suliman ME, Divino Filho JC, Barany P, Anderstam B, Lindholm B, Bergstrom J. Effects of high-dose folic acid and pyridoxine on plasma and erythrocyte sulfur amino acids in hemodialysis patients. J. Am. Soc. Nephrol. 10(6), 1287-1296 (1999).

30 Agli AN, Schaefer A, Geny B, Piquard F, Haberey P. Erythrocytes participate significantly in blood transport of amino acids during the post absorptive state in normal humans. Eur. J. Appl. Physiol. Occup. Physiol. 78(6), 502-508 (1998).

31 Schaefer A, Piquard F, Haberey P. The effects of changes in plasma amino acid concentrations on erythrocyte amino acid content. Clin. Biochem. 23(3), 237-240 (1990).

32 Han SS, Sue LY, Berndt SI et al. Associations between genes in the one-carbon metabolism pathway and advanced colorectal adenoma risk in individuals with low folate intake. Cancer Epidemiol. Biomarkers Prev. 21(3), 417-427 (2012).

33 Struys EA, Jansen E, De Meer K, Jakobs C. Determination of $S$-adenosylmethionine and $S$-adenosylhomocysteine in plasma and cerebrospinal fluid by stable-isotope dilution tandem mass spectrometry. Clin. Chem. 46(10), 1650-1656 (2000). 